\title{
Discrete Pseudo Almost Periodic Solutions for Some Difference Equations
}

\author{
Elhadi Ait Dads", Khalil Ezzinbi, Lahcen Lhachimi \\ University Cadi Ayyad, Faculty of Sciences Semlalia, Department of Mathematics, Marrakesh, Morocco \\ E-mail:"eaitdads@gmail.com,ezzinbi@ucam.ac.ma,lllahcen@gmail.com \\ Received February 23, 2011; revised April 26, 2011; accepted May 10, 2011
}

\begin{abstract}
In this work, we study the existence and uniqueness of pseudo almost periodic solutions for some difference equations. Firstly, we investigate the spectrum of the shift operator on the space of pseudo almost periodic sequences to show the main results of this work. For the illustration, some applications are provided for a second order differential equation with piecewise constant arguments.
\end{abstract}

Keywords: Difference Equations, Pseudo Almost Periodic Sequences, Schift Operator

\section{Introduction}

Difference equations have many applications in populations dynamics, they are used to describe the evolution of many phenomena over the course of time. For example, if a certain population has discrete generations, the size of the $(n+1)$ th generation $x(n+1)$ is a function of the $n$th generation $x(n)$. This relation expresses itself in the following difference equation

$$
x(n+1)=f(x(n)), n \in \mathbb{Z} .
$$

The discrete processes occur in the investigation of many phenomena, mainly in the case of use of computers. One of the most widely adopted definition of a discrete process can be formulated as follows: a discrete process is a map from the additive group of the integers $\mathbb{Z}$, into a complete metric space $(X, d)$, such as $\mathbb{R}^{m}$ or $\mathbb{C}^{m}$ with the distance function induced by the vector norm.

We use two different notations to designate a discrete process, namely, if $f: \mathbb{Z} \rightarrow X$ is a discrete process, we shall write instead $\{f(n)\}_{n \in \mathbb{Z}}$ or $\left\{f_{n}\right\}_{n \in \mathbb{Z}}$, dropping usually the subscript " $n \in \mathbb{Z}$ ”, since no confusion can occur (indeed, we are not going to consider in this work discrete processes defined on a group, other than $\mathbb{Z}$ ).

Of course, one of the most common sources for the discrete processes is the theory of difference equations, such as

$$
x_{n+1}=A x_{n}+b_{n}, n \in \mathbb{Z},
$$

where $x_{n}$ stands for the unknown process, with values in $\mathbb{R}^{m}$ or $\mathbb{C}^{m} A$ is a square matrix of order $m$ with real or complex entries and $b_{n}$ stands for a given discrete process, with values in the same space as $\left(x_{n}\right)$. In practice, we deal with solutions of (2) which are only defined on subsets of $\mathbb{Z}$, and therefore, they might be regarded as restrictions of a "complete" process to a subset of its domain of definition.

Difference equations and discrete dynamic systems represent two sides of the same coin. For instance, when mathematicians talk about difference equations, they usually refer to the analytic theory of the subject, and when they talk about discrete dynamic systems, they generally refer to its geometrical and topological aspects.

More sophisticated equations (or systems) than (2) are those described by the following discrete equation

$$
x_{n+1}=f\left(n, x_{n}\right), n \in \mathbb{Z}
$$

where $f: \mathbb{Z} \times \mathbb{R}^{m} \rightarrow \mathbb{R}^{m}$ (or $\mathbb{C}^{m}$ ) is a given map, in general nonlinear in both arguments.

Another example, let $y(n)$ be the size of a population at time $n$. If $\mu$ is the rate of growth of the population from one generation to another, then we may consider a mathematical model in the form

$$
y(n+1)=\mu y(n), \mu>0 .
$$

If the initial population is given by $y(0)=y_{0}$, then the solutions are given by $y(n)=\mu^{n} y_{0}$. If $\mu>1$, then $y(n)$ increases infinitely, and $\lim _{n \rightarrow \infty} y(n)=\infty$. If $\mu=1$, then $y(n)=y_{0}$ for all $n>0$, which means that the size of the population is constant for the indefinite future. 
However, for $\mu<1$, we have $\lim _{n \rightarrow \infty} y(n)=0$, and the population eventually becomes extinct.

Since our main objective is to provide a criteria to get the existence of a pseudo almost periodic solution for equations of the form (2) or (3), we shall first review the basic properties of pseudo almost periodic discrete processes.

This work is motivated by the results obtained in [1,2], and the main results would be some extension for some well-established results in the literature, more details can be found in [3].

This work is organized as follows. In Section 2, we consider geometrical properties of the shift operator in general case and, we deal with the properties of shift operator the spaces of almost periodic and on ergodic sequences. In Section 3, we a consider the existence and uniqueness solutions of some difference equations using polynomial functions. In the last section, we deal with the application of the previous results to some second order differential equation with a piecewise constant argument.

\section{Shift Operator Acting on the Space of Pseudo Almost Periodic Sequences}

In this section, we give some properties on pseudo almost periodic sequences that will be used in this work. For more details in this connexion, the reader will see [4-12].

Definition 2.1: A sequence $\left(x_{n}\right)_{n \in \mathbb{Z}}$ with values in $\mathbb{C}^{m}$ is called almost periodic if for all $\varepsilon>0$, the set $T(x, \varepsilon):=\left\{\tau \in \mathbb{Z}:\right.$ for all $\left.n \in \mathbb{Z},\left\|x_{n+\tau}-x_{n}\right\|<\varepsilon\right\}$ is relatively dense.

The space of almost periodic sequences is denoted by $A P\left(\mathbb{Z}, \mathbb{C}^{m}\right)$. If $m=1$, we use the notation $A P(\mathbb{Z})$. Let $\left(B,\|\cdot\|_{\infty}\right)$ denote the space of bounded complex sequences provide with the supremum norm. $P A P_{0}(\mathbb{Z})$ denote the space of bounded complex sequences $\left(x_{n}\right)_{n \in \mathbb{Z}}$ satisfying the ergodicity condition

$$
\lim _{N \rightarrow+\infty} \frac{1}{2 N} \sum_{n=-N}^{N}\left|x_{n}\right|=0 .
$$

Remark 2.2: $\lim _{N \rightarrow+\infty} \frac{1}{2 N} \sum_{n=-N}^{N}\left|x_{n}\right|=0$, doesn't imply that $\left(x_{n}\right)_{n \in \mathbb{Z}}$ is bounded. In fact, let us consider the sequence defined by

$$
x_{n}=\left\{\begin{array}{l}
p \text { if } n=p^{3} \\
0 \text { otherwise. }
\end{array}\right.
$$

Let $p \in \mathbb{N}$ be such that $p^{3} \leq N<(p+1)^{3}$. Then

$$
\frac{1}{2 N} \sum_{n=-N}^{N}\left|x_{n}\right|=\frac{1}{N} \sum_{k=1}^{p} k \leq \frac{p(p+1)}{2 p^{3}} \underset{p \rightarrow \infty}{\rightarrow} 0 .
$$

For a function $f: \mathbb{R} \rightarrow \mathbb{C}^{m}$, we define $T(f, \varepsilon)$ by

$$
T(f, \varepsilon)=\{\tau \in \mathbb{R}: \text { for all } t \in \mathbb{R},|f(t+\tau)-f(t)|<\varepsilon\} .
$$

Definition 2.3: A bounded continuous function $x$ is said to be almost periodic if the set $T(f, \varepsilon)$ is relatively dense for all $\varepsilon>0$.

For the next $A P\left(\mathbb{R}, \mathbb{C}^{m}\right)$ denotes the space of all almost periodic functions from $\mathbb{R}$ to $\mathbb{C}^{m}$.

Proposition 2.4: Let $m \in \mathbb{N}^{*}$ and $x=\left(x_{n}\right)_{n \in \mathbb{Z}}$ be $a$ sequence with values in $\mathbb{C}^{m}$. Let define the function $\bar{x}$ : $\mathbb{R} \rightarrow \mathbb{C}^{m}$ by

$$
\bar{x}(n)=x_{n} \text { for all } n \in \mathbb{Z},
$$

and $\bar{x}$ is affine in $[n, n+1]$. Then the following results are true.

1) $\sup _{t \in \mathbb{R}}\|\bar{x}(t)\|=\sup _{n \in \mathbb{Z}}\left\|x_{n}\right\|, \quad T(\bar{x}, \varepsilon) \cap \mathbb{Z} T(x, \varepsilon)$ and $\bar{x} \in A P\left(\mathbb{R}, \mathbb{C}^{m}\right)$ if and only if $x \in A P\left(\mathbb{Z}, \mathbb{C}^{m}\right)$,

2) $x \in P A P_{0}\left(\mathbb{Z}, \mathbb{C}^{m}\right)$ if and only if $\bar{x} \in P A P\left(\mathbb{R}, \mathbb{C}^{m}\right)$.

Proof. 1) is a consequence of results taken from [1].

For the proof of 2), by taking the components, real part and imaginary part, we can consider the case where $x \in P A P_{0}(\mathbb{Z}, \mathbb{R})$. Then, one has

For $t \in[n, n+1]$ one has

$\bar{x}(t)=\left(x_{n+1}-x_{n}\right)(t-n)+x_{n}=x_{n+1}(t-n)+x_{n}(n+1-t)$

Two cases to be considered:

a) If $x_{n} x_{n+1} \geq 0$

$$
\int_{n}^{n+1}|\bar{x}(t)| \mathrm{d} t=\frac{\left|x_{n+1}\right|+\left|x_{n}\right|}{2} .
$$

b) If $x_{n} x_{n+1}<0$

$$
\begin{aligned}
& \quad \frac{\left|x_{n+1}\right|+\left|x_{n}\right|}{2} \leq \frac{x_{n}^{2}+x_{n+1}^{2}}{2\left(\left|x_{n+1}\right|+\left|x_{n}\right|\right)}=\int_{n}^{n+1}|\bar{x}(t)| \mathrm{d} t \\
& \leq \frac{\left|x_{n+1}-x_{n}\right|}{2}+\left|x_{n}\right| \leq \frac{\left|x_{n+1}\right|+3\left|x_{n}\right|}{2} .
\end{aligned}
$$

The result is a consequence of the fact that $\bar{x} \in$ $P A P_{0}(\mathbb{R}, \mathbb{R})$ if and only if $\left(\int_{n}^{n+1}|\bar{x}(t)| \mathrm{d} t\right)_{n \in \mathbb{Z}} \in P A P_{0}(\mathbb{R}, \mathbb{Z})$.

Definition 2.5: We define the space of pseudo almost periodic sequences by

$$
P A P(\mathbb{Z})=A P(\mathbb{Z}) \oplus P A P_{0}(\mathbb{Z}) .
$$

Proposition 2.6: [2] Let $x \in P A P(\mathbb{Z})$ be such that 
$x=y+z$, for some $y \in A P(\mathbb{Z})$ and $z \in P A P_{0}(\mathbb{Z})$ Then $\|y\|_{\infty} \leq\|x\|_{\infty}$.

Difference calculus is the discrete analogue of the familiar differential and integral calculus. In this section, we introduce some basic properties of two the following operators that are essential in difference equations

$$
\Delta x(n)=x(n+1)-x(n)
$$

and the shift operator

$$
\operatorname{Ex}(n)=x(n+1) .
$$

Then

$$
E^{k} x(n)=x(n+k) .
$$

Let $I$ be the identity operator. Then $\Delta=E-I$ and $E=\Delta+I$. The following formula are true

$$
\begin{aligned}
\Delta^{k} x(n)=(E-I)^{k} x(n) & =\sum_{i=0}^{k}(-1)^{i}\left(\begin{array}{c}
k \\
i
\end{array}\right) E^{k-i} x(n) \\
& =\sum_{i=0}^{k}(-1)^{i}\left(\begin{array}{c}
k \\
i
\end{array}\right) x(n+k-i) .
\end{aligned}
$$

Similarly we have

$$
E^{k} x(n)=\sum_{i=0}^{k}\left(\begin{array}{l}
k \\
i
\end{array}\right) \Delta^{k-i} x(n) .
$$

We should point out here that the operator $\Delta$ is the counterpart of the derivative operator $D$ in calculus. Both operators $E$ and $\Delta$ share one of the helpful features of the derivative operator $D$, namely, the property of linearity. Another interesting difference, parallel to differential calculus, is the discrete analogue of the fundamental theorem of calculus.

Remark 2.7: Exponential exp at in differential equations corresponds to the exponential $a^{n}$ and the integral $\int_{0}^{t} \exp a(t-s) g(s) \mathrm{d} s$ corresponds to the summation: $\sum_{k=0}^{n-1} a^{n-k-1} g(k)$.

Let us consider the linear map defined by

$$
\left\{\begin{array}{l}
T: \mathbb{C}^{\mathbb{Z}} \rightarrow \mathbb{C}^{\mathbb{Z}} \\
\left(x_{n}\right)_{n \in \mathbb{Z}} \rightarrow\left(x_{n}+1\right)_{n \in \mathbb{Z}} .
\end{array}\right.
$$

Let $F$ be a subspace of $B$ that is invariant by $T$, for example $F$ could be one of the following spaces $A P(\mathbb{Z}, \mathbb{C}), \quad P A P_{0}(\mathbb{Z}, \mathbb{C}), \quad P A P(\mathbb{Z}, \mathbb{C})$. Let $T_{F}$ be the linear map induced by $T$ on $F$ and take $y \in F$ and $P \in \mathbb{C}[X]$, where $\mathbb{C}[X]$ is the space of polynomial functions over $\mathbb{C}$. Next, we study the existence of solutions in $F$ for a given $y \in F$ for the following algebraic equation

$$
P\left(T_{F}\right) x=y .
$$

This equation has solutions if $y \in \operatorname{Im} P\left(T_{F}\right)$, but we have to compute $\operatorname{Im} P\left(T_{F}\right)$. The uniqueness problem is equivalent to determine $\operatorname{ker} P\left(T_{F}\right)$. The following result is well-established.

Lemma 2.8: Let $P \in \mathbb{C}[X]$ be non constant. Then

$$
\operatorname{ker} P(T)=\left\{\left(\sum_{i=1}^{N} \lambda_{i}^{n} Q_{i}(n)\right)_{n \in \mathbb{Z}}: \text { with } \operatorname{deg}\left(Q_{i}\right)<m_{i}\right\}
$$

where the $\lambda_{i}^{\prime} s$ are non zero roots of $P$ with respective multiplicities $m_{i}$.

Remark 2.9: If 0 is the unique root of $P$, then $\operatorname{ker} P(T)=\{0\}$.

Lemma 2.10: Let $T_{1}=T_{\mid B}$ (the restriction of $T$ to B). Then

$$
\operatorname{ker} P\left(T_{1}\right)=\operatorname{vect}\left\{\left(\lambda_{i}^{n}\right)_{n \in \mathbb{Z}},\left|\lambda_{i}\right|=1, i=1, \cdots, r\right\} .
$$
Then

Proof. Let $\lambda$ be a complex number such that $|\lambda|=1$.

$$
\operatorname{ker}\left(T_{1}-\lambda I\right)=\left\{\left(c \lambda^{n}\right)_{n \in \mathbb{Z}}, c \in \mathbb{C}\right\} .
$$

Let $y \in \operatorname{ker}\left(T_{1}-\lambda I\right)^{2}$ and $x=\left(T_{B}-\lambda I\right)(y)$. Then

$$
x_{n+1}-\lambda x_{n}=0 \text { and } y_{n+1}-\lambda y_{n}=x_{n},
$$

which implies that $y_{n+1}-\lambda y_{n}=\lambda^{n} x_{0}$, and

$$
\frac{y_{n+1}}{\lambda^{n+1}}-\frac{y_{n}}{\lambda^{n}}=\frac{x_{0}}{\lambda} \text {. }
$$

Since $\frac{y_{n}}{\lambda^{n}}$ is also bounded, because $|\lambda|=1$, then $x_{0}=0$ and $y_{n+1}=\lambda y_{n}$, also

$$
\operatorname{ker}\left(T_{1}-\lambda I\right)^{2}=\operatorname{ker}\left(T_{1}-\lambda I\right) \text {. }
$$

By simple recurrence on $m$, we deduce that for all $m \geq 1$,

$$
\operatorname{ker}\left(T_{1}-\lambda I\right)^{m}=\operatorname{ker}\left(T_{1}-\lambda I\right)=\left\{\left(c \lambda^{n}\right)_{n \in \mathbb{Z}}: c \in \mathbb{C}\right\} .
$$

Consequently:

$$
\begin{aligned}
\operatorname{ker} P\left(T_{1}\right) & =\underset{1 \leq i \leq r}{\oplus} \operatorname{ker}\left(T_{1}-\lambda_{i} I\right)^{m_{i}} \\
& =\operatorname{vect}\left\{\left(\lambda_{i}^{n}\right)_{n \in \mathbb{Z}}:\left|\lambda_{i}\right|=1, i=1, \cdots, r\right\} .
\end{aligned}
$$

Lemma 2.11: Let $x, y \in \mathbb{C}^{\mathbb{Z}}$. Consider $x * y$ the sequence defined by

$$
(x * y)_{n}= \begin{cases}\sum_{0 \leq k \leq n-1} x_{k} y_{n-1-k} & \text { if } n>0 \\ 0 & \text { if } n=0 . \\ -\sum_{n \leq k \leq-1} x_{k} y_{n-1-k} & \text { if } n<0 .\end{cases}
$$


Then $(x, y) \rightarrow x * y$ is bilinear and symmetric. Moreover, if we denote by $\Delta_{\lambda}=T-\lambda I$, then

$$
\Delta_{\lambda}(x * y)=x_{0} y+\left(\Delta_{\lambda} x\right) * y
$$

Remark 2.12: If $x \in \mathbb{C}^{\mathbb{Z}}$, then $x$ can be extended to a function $\tilde{x}$ which is of stepping type on $\mathbb{R}$ in the following manner: $\tilde{x}(t)=x(E(t))$ where $E(t)$ denotes the greatest integer function of $t$, then one has for $x, y \in \mathbb{C}^{\mathbb{Z}}$,

$$
(x * y)(n)=\int_{0}^{n} \tilde{x}(t) \tilde{y}(n-t) \mathrm{d} t .
$$

Proof. Using the above remark, one can see that the following map $(x * y) \rightarrow x * y$ is bilinear and symmetric. On the other hand, one has

$$
\begin{aligned}
\Delta_{\lambda}(x * y)(n)= & \int_{0}^{n+1} \tilde{x}(t) \tilde{y}(n+1-t) \mathrm{d} t-\lambda \int_{0}^{n} \tilde{x}(t) \tilde{y}(n-t) \mathrm{d} t \\
= & \int_{0}^{1} \tilde{x}(t) \tilde{y}(n+1-t) \mathrm{d} t \\
& +\int_{1}^{n+1} \tilde{x}(t) \tilde{y}(n+1-t) \mathrm{d} t-\lambda \int_{0}^{n} \tilde{x}(t) \tilde{y}(n-t) \mathrm{d} t \\
= & x_{0} y_{n}+\int_{0}^{n} \tilde{x}(u+1) \tilde{y}(n-u) \mathrm{d} u \\
& -\lambda \int_{0}^{n} \tilde{x}(t) \tilde{y}(n-t) \mathrm{d} t \\
= & x_{0} y_{n}+\int_{0}^{n}(\tilde{x}(t+1)-\lambda \tilde{x}(t)) \tilde{y}(n-t) \mathrm{d} t \\
= & x_{0} y_{n}+\left(\left(\Delta_{\lambda} x\right) * y\right)(n)
\end{aligned}
$$

In the sequel, we denote by

$$
\begin{aligned}
& F_{p, \lambda}=\left\{\left(b_{n}+\lambda^{n} Q(n)\right)_{n \in \mathbb{Z}},\right. \text { such that } \\
&\left.\left(b_{n}\right)_{n \in \mathbb{Z}} \in F \text { and deg } Q \leq p\right\} .
\end{aligned}
$$

We define the following polynomials

$$
C_{X}^{0}=\text { 1and } C_{X}^{p}=\frac{X(X-1) \cdots(X-p+1)}{p !} \text { if } p \in \mathbb{N}^{*} \text {. }
$$

Lemma 2.13: Let $y \in \mathbb{C}^{\mathbb{Z}}$ and $\lambda$ be a complex number such that $|\lambda|=1$, Then for $p \in \mathbb{N}$, the following are true

1) $\left(\Delta_{\lambda}\right)^{p+1}\left(c_{p, \lambda} * y\right)=y$ where $c_{p, \lambda}=\left(C_{n}^{p} \lambda^{n-p}\right)_{n \in \mathbb{Z}}$.

2) $\operatorname{Im}\left(T_{F}-\lambda I\right)^{p+1}=\{y \in F$ : such that $\left.c_{p, \lambda} * y \in F_{p, \lambda}\right\}$.

Proof. 1) For $p>0$, we claim that

$$
\Delta_{\lambda}\left(c_{p, \lambda} * y\right)=c_{p-1, \lambda} * y .
$$

From lemma (2.11) one has :

$$
\begin{aligned}
\Delta_{\lambda}\left(c_{p, \lambda} * y\right) & =\left(c_{p, \lambda}\right)_{0} y+\Delta_{\lambda}\left(c_{p, \lambda}\right) * y \\
& =\Delta_{\lambda}\left(c_{p, \lambda}\right) * y=c_{p-1, \lambda} * y .
\end{aligned}
$$

It follows that

for all $p \geq 0$

$$
\begin{aligned}
\Delta_{\lambda}^{p+1}\left(c_{p, \lambda} * y\right) & =\Delta_{\lambda}\left(c_{0, \lambda} * y\right) \\
& =\left(c_{0, \lambda}\right)_{0} y+\left(\Delta_{\lambda} c_{0, \lambda}\right) * y=y .
\end{aligned}
$$

2) One has $y \in \operatorname{Im}\left(T_{F}-\lambda\right)^{p+1}$ if and only if there exists $x \in F$ such that $\left(T_{F}-\lambda\right)^{p+1}(x)=y$. From lemma (2.8) and 1) one has: $x_{n}=\lambda^{n} Q(n)+\left(c_{p, \lambda} * y\right)_{n}$ is in $F$ if and only if $c_{p, \lambda} * y$ is in $F_{p, \lambda}$.

Proposition 2.14: Let $y \in \mathbb{C}^{\mathbb{Z}}, \lambda$ be a complex number such that $|\lambda|=1$, and $Q$ be a polynomial of degree $p$. Then the following are true

$$
\begin{aligned}
& \operatorname{Im}\left(T_{F}-\lambda I\right)^{p+1} \\
& =\left\{y \in F, \text { such that }\left(Q(n) \lambda^{n}\right)_{n \in \mathbb{Z}} * y \in F_{p, \lambda}\right\} .
\end{aligned}
$$

In particular

$\operatorname{Im}\left(T_{F}-\lambda I\right)^{p+1}=\left\{y \in F\right.$, such that $\left.\left(n^{p} \lambda^{n}\right)_{n \in \mathbb{Z}} * y \in F_{p, \lambda}\right\}$.

and

$$
\begin{aligned}
& \operatorname{Im} P\left(T_{F}\right)=\{y \in F: \text { such that for all } i \in[1, r], \\
& \left.\left(n^{m_{i}-1} \lambda_{i}^{n}\right)_{n \in \mathbb{Z}} * y \in F_{m_{i}-1, \lambda_{i}}\right\}
\end{aligned}
$$

Proof. Let $y \in \operatorname{Im}\left(T_{F}-\lambda I\right)^{p+1}$. Then for all $q \leq p$, $y \in \operatorname{Im}\left(T_{F}-\lambda I\right)^{q+1}$, by lemma (2.13) we have

For all $q \leq p, \quad\left(C_{n}^{q} \lambda^{n-q}\right)_{n \in \mathbb{Z}} * y$ is in $F_{q, \lambda}$, which is equivalent to

For all $q \leq p,\left(C_{n}^{q} \lambda^{n}\right)_{n \in \mathbb{Z}} * y$ is in $F_{q, \lambda}$, and as $\left(C_{X}^{q}\right)_{0 \leq q \leq p}$ is a basis of $\mathbb{C}_{p}[X]$, then

$$
\left(Q(n) \lambda^{n}\right)_{n \in \mathbb{Z}} * y \text { is in } F_{p, \lambda} .
$$

Conversely, assume that $\left(Q(n) \lambda^{n}\right)_{n \in \mathbb{Z}} * y$ is in $F_{p, \lambda}$. One has from lemma (2.11)

$$
\begin{aligned}
& x * y \text { and } y \text { are in } F_{p, \lambda} \text { which implies that } \\
& \qquad\left(\Delta_{\lambda} x\right) * y \text { is in } F_{p, \lambda} .
\end{aligned}
$$

But

$$
\Delta_{\lambda}\left(Q(n) \lambda^{n}\right)_{n \in \mathbb{Z}}=\left(\left(\Delta_{1} Q\right)(n) \lambda^{n+1}\right)_{n \in \mathbb{Z}},
$$

then

$$
\left(\left(\Delta_{1} Q\right)(n) \lambda^{n}\right)_{n \in \mathbb{Z}} * y \text { is in } F_{p, \lambda},
$$

by iteration, we see that

$$
\text { for all } q \in[0, p],\left(\left(\Delta_{1}^{q} Q\right)(n) \lambda^{n}\right)_{n \in \mathbb{Z}} * y \text { is in } F_{p, \lambda},
$$


and since $\left(\Delta_{1}^{q} Q\right)_{0 \leq q \leq p}(X)$ is a basis of $\mathbb{C}_{p}[X]$, then for all $R \in \mathbb{C}_{p}[X],\left(R(n) \lambda^{n}\right)_{n \in \mathbb{Z}} * y$ is in $F_{p, \lambda}$,

In particular

$$
\left(C_{n}^{p} \lambda^{n-p}\right)_{n \in \mathbb{Z}} * y \text { is in } F_{p, \lambda},
$$

and consequently $y \in \operatorname{Im}\left(T_{F}-\lambda I\right)^{p+1}$. The end of the proof results from the fact that

$$
\operatorname{Im} P\left(T_{F}\right)=\underset{1 \leq i \leq r}{\cap} \operatorname{Im}\left(T_{F}-\lambda_{i} I\right)^{m_{i}} .
$$

Let $\tau$ be the linear map induced by $T$ on $A P(\mathbb{Z}, \mathbb{C})$

It is clear that if $|\lambda| \neq 1$, then $(\tau-\lambda I)$ is invertible. On the other hand if the roots of $P$ are of modulus different from 1 , then $P(\tau)$ is invertible, in this case ker $P(\tau)=\{0\}$ and we have the uniqueness of the solutions. Let $\left(\lambda_{i}\right)_{1 \leq i \leq r}$ be the roots of $P$ with modulus 1. Then

$$
P(X)=\prod_{1 \leq i \leq r}\left(X-\lambda_{i}\right)^{m_{i}} Q(X)
$$

where $Q$ is a polynomial whose roots are of modulus different from 1 .

\section{Proposition 2.15:}

$$
\operatorname{ker} P(\tau)=\operatorname{vect}\left\{\left(\lambda_{i}^{n}\right)_{n \in \mathbb{Z}}:\left|\lambda_{i}\right|=1, i=1, \cdots, r\right\} \text {. }
$$

Proof. From lemma (2.10), one has

$$
\begin{aligned}
\operatorname{ker} P(\tau) & =A P(\mathbb{Z}, \mathbb{C}) \cap \operatorname{vect}\left\{\left(\lambda_{i}^{n}\right)_{n \in \mathbb{Z}},\left|\lambda_{i}\right|=1, i=1, \cdots, r\right\} \\
& =\operatorname{vect}\left\{\left(\lambda_{i}^{n}\right)_{n \in \mathbb{Z}},\left|\lambda_{i}\right|=1 \text {, for } i=1, \ldots, r\right\},
\end{aligned}
$$

(since $\left(\lambda_{i}^{n}\right)_{n \in \mathbb{Z}}$ is periodic).

It is well known that if $Q_{1}, Q_{2} \in \mathbb{C}[X]$ and $Q_{1} \wedge Q_{2}=1$, then

$$
\operatorname{Im}\left(Q_{1} Q_{2}\right)(\tau)=\operatorname{Im} Q_{1}(\tau) \cap \operatorname{Im} Q_{2}(\tau),
$$

and

$$
\operatorname{Im} P(\tau)=\bigcap_{1 \leq i \leq r} \operatorname{Im}\left(\tau-\lambda_{i} I\right)^{m_{i}}
$$

\section{Existence of Pseudo Almost Periodic Sequences}

It is known that if $A \in M_{n}(\mathbb{C}), \quad b \in A P\left(\mathbb{Z}, \mathbb{C}^{m}\right)$ and $x$ is a bounded solution of

$$
x_{n+1}=A x_{n}+b_{n}, n \in \mathbb{Z}
$$

then $x$ is almost periodic.

If $y \in A P(\mathbb{Z}, \mathbb{C})$ and $P \in \mathbb{C}[X]$, by transforming the scalar equation

$$
P P(\tau) x=y,
$$

to a system, we deduce the following lemma.

Lemma 3.1: For $y \in A P(\mathbb{Z}, \mathbb{C})$ and $P \in \mathbb{C}[X]$, every bounded solution of $P(T)(x)=y$ is almost periodic.

Consequently from lemma (3.1) and the proposition (2.14), we get the following result.

Proposition 3.2: Let $\lambda$ be a complex number such that $\lambda \neq 0, p \in \mathbb{N}$ and $Q$ be a polynomial with degree p. Then

$$
\begin{aligned}
& \operatorname{Im}(\tau-\lambda I)^{p+1} \\
& =\left\{y \in A P(\mathbb{Z}, \mathbb{C}), \text { such that }\left(Q(n) \lambda^{n}\right)_{n \in \mathbb{Z}} * y \in B_{p, \lambda}\right\} .
\end{aligned}
$$

In particular

$$
\begin{aligned}
& \operatorname{Im}(\tau-\lambda I)^{p+1} \\
& =\left\{y \in A P(\mathbb{Z}, \mathbb{C}), \text { such that }\left(n^{p} \lambda^{n}\right)_{n \in \mathbb{Z}} * y \in B_{p, \lambda}\right\}^{.}
\end{aligned}
$$

and

$\operatorname{Im} P(\tau)=\{y \in A P(\mathbb{Z}, \mathbb{C})$, such that for all $i \in[1, r]$,

$\left.\left(n^{m_{i}-1} \lambda_{i}^{n}\right)_{n \in \mathbb{Z}} * y \in B_{m_{i}-1, \lambda_{i}}\right\}$.

In the next, we are concerned with the solutions in $A P(\mathbb{Z}, \mathbb{C})$ of the following equation

$$
(\tau-I)^{p+1}(x)=y \text {, for } p \in \mathbb{N} .
$$

Proposition 3.3: Let $y \in \mathbb{C}^{\mathbb{Z}}$. Then we have

1) $(T-I)^{p+1}\left(y * c_{p}\right)=y,\left(\right.$ where $\left.c_{p}=\left(C_{n}^{p}\right)_{n \in \mathbb{Z}}\right)$,

2) $y \in \operatorname{Im}(\tau-I)^{p+1}$ if and only if there exists $b \in B$ such that $y * c_{p}=b+Q$ with $Q \in \mathbb{C}_{p}[X]$.

3) the solutions of $(\tau-I)^{p+1}(x)=y$ in $A P(\mathbb{Z}, \mathbb{C})$ are $x_{n}=b_{n}+c$ where $c$ is a constant and

$$
\left\{\begin{array}{l}
b=y * c_{p}-\sum_{0 \leq k \leq p} \alpha_{k} c_{k} \\
\alpha_{p}=\lim _{n \rightarrow+\infty} \frac{\left(y * c_{1}\right)(n)}{n} \\
\alpha_{k+1}=\lim _{n \rightarrow+\infty} \frac{\left(y * c_{1}\right)(n)-\left(\alpha_{k+2} C_{n}^{2}+\cdots+\alpha_{p} C_{n}^{p-k}\right)}{n} .
\end{array}\right.
$$

Proof. Since $\left(C_{X}^{k}\right)_{0 \leq k \leq p}$ is a basis of $\mathbb{C}_{p}[X]$, then there exit scalars $\left(\alpha_{k}\right)_{0 \leq k \leq p}$ such that $Q=\sum_{0 \leq k \leq p} \alpha_{k} c_{k}$ in this case, one has

$$
y * c_{p}=b+\sum_{0 \leq k \leq p} \alpha_{k} c_{k} .
$$


Then, by applying $(T-I)^{p-1}$ to the equation (7), we obtain

$$
y * c_{1}=(T-I)^{p-1}(b)+\alpha_{p-1}+\alpha_{p} c_{1},
$$

then

$$
\alpha_{p}=\lim _{n \rightarrow+\infty} \frac{\left(y * c_{1}\right)(n)}{n} .
$$

Suppose that $\alpha_{p}, \alpha_{p-1}, \cdots, \alpha_{k+2}$ are known and let us compute $\alpha_{k+1}$ : by applying $(T-I)^{k}$ to the equation (7), one has

$y * c_{p-k}=(T-I)^{k}(b)+\alpha_{k}+\alpha_{k+1} c_{1}+\alpha_{k+2} c_{2}+\cdots+\alpha_{p} c_{p-k}$,

so

$\alpha_{k+1}=\lim _{n \rightarrow+\infty} \frac{\left(y * c_{p-k}\right)(n)-\left(\alpha_{k+2} C_{n}^{2}+\cdots+\alpha_{p} C_{n}^{p-k}\right)}{n}$.

We conclude that for all $y \in A P(\mathbb{Z}, \mathbb{C})$, the equation $(\tau-I)^{p+1}(x)=y$ admits the solutions in $A P(\mathbb{Z}, \mathbb{C})$ if and only if, the limits given by equations (8) and (9) exist and the sequence $y * c_{p}-\sum_{0 \leq k \leq p} \alpha_{k} c_{k}$ is bounded, in this case the solutions are given by

$$
x=y * c_{p}-\sum_{0 \leq k \leq p} \alpha_{k} c_{k},
$$

with $\alpha_{0}$ is any constant and the $\left(\alpha_{k}\right)_{1 \leq k \leq p}$ are given by (8) and (9).

Remark 3.4: By a change of variables, the equation $(\tau-\lambda I)^{p+1}(x)=y$ when $|\lambda|=1$ becomes in the previous form (6).

Indeed, let us consider the following operator

$$
\begin{aligned}
M_{\lambda}: A P(\mathbb{Z}, \mathbb{C}) & \rightarrow A P(\mathbb{Z}, \mathbb{C}) \\
x & \rightarrow\left(\lambda^{n} x_{n}\right)_{n \in \mathbb{Z}} .
\end{aligned}
$$

Since $\tau M_{\lambda}=\lambda M_{\lambda} \tau$, then

$$
(\tau-\lambda I) M_{\lambda}=\lambda M_{\lambda} \tau-\lambda M_{\lambda}=\lambda M_{\lambda}(\tau-I) .
$$

So

$$
\tau-\lambda I=\lambda M_{\lambda}(\tau-I) M_{\lambda}^{-1},
$$

and

$$
(\tau-\lambda I)^{p+1}=\lambda^{p+1} M_{\lambda}(\tau-I)^{p+1} M_{\lambda}^{-1} .
$$

Then equation

$$
(\tau-\lambda I)^{p+1}(x)=y,
$$

becomes

$$
\lambda^{p+1} M_{\lambda}(\tau-I)^{p+1} M_{\lambda}^{-1}(x)=y,
$$

differently

$$
(\tau-I)^{p+1} M_{\lambda^{-1}}(x)=\frac{1}{\lambda^{p+1}} M_{\lambda^{-1}} y,
$$

then by putting $X=M_{\lambda^{-1}}(x)$ and $Y=\frac{1}{\lambda^{p+1}} M_{\lambda^{-1}} y$, and we are coming down to the following equation $(\tau-I)^{p+1}(X)=Y$.

Theorem 3.5 For the general equation $P(\tau) x=y$, the solution is of the form: $x=x_{0}+x_{1}$, such that $x_{1}=\sum_{i=1}^{r} x_{i}$, (with $r$ is the number of different roots with modulus equal to $1, x_{i}$ is the solution of the equation $\left.\left(\tau-\lambda_{i} I\right)^{p_{i}+1}\left(x_{i}\right)=y_{i}\right)$ and $x_{0}$ an element of $\operatorname{ker} P(\tau)$.

Proof. We write $P$ under the form

$$
P=\prod_{i=1}^{r}\left(X-\lambda_{i}\right)^{1+p_{i}} Q
$$

with $\lambda_{i}^{\prime} s$ are the roots of $P$ which are of modulus equal to 1 and $p_{i} \in \mathbb{N}$, Even if it means to replace $y$ by $Q(\tau)^{-1} y$, we may come down to $Q=1$. Indeed let us putting $P=\prod_{k=1, k \neq i}^{r}\left(X-\lambda_{k}\right)^{1+p_{k}}$, then using the Bezout identity, we get that there exist polynomials $U_{i}$ such that $\sum_{i=1}^{r} A_{i} U_{i}=1$. Then $y=\sum_{i=1}^{r} A_{i}(\tau) y_{i} \quad$ with $y_{i}=U_{i}(\tau) y$ and equation

$$
P(\tau)(x)=y
$$

becomes

$$
P(\tau)(x)=\sum_{i=1}^{r} A_{i}(\tau) y_{i}
$$

then to built a solution of Equation (12), it suffices to determine a solution $x_{i}$ of the following equation

$$
\left(\tau-\lambda_{i} I\right)^{p_{i}+1}\left(x_{i}\right)=y_{i},
$$

the solution is easily determined and after we take $x=\sum_{i=1}^{r} x_{i}$. To obtain all solutions, we add elements of

$$
\operatorname{ker} P(\tau)=\operatorname{vect}\left\{\left(\lambda_{i}^{n}\right)_{n \in \mathbb{Z}}, 1 \leq i \leq r\right\} .
$$

Example 3.6: For all polynomial $Q$ with all roots are of modulus different from 1 , in $\mathbb{C}[X]$ one has the following decomposition $\frac{1}{Q}=\sum_{i=1}^{r} \sum_{j=1}^{m_{i}} \frac{a_{i, j}}{\left(X-\lambda_{i}\right)^{j}} \quad$ where the $\lambda_{i}^{\prime} s$ are two by two distinct and of modulus different from 1 , then we have 
$Q(\tau)^{-1}=\sum_{i=1}^{r} \sum_{j=1}^{m_{i}} a_{i, j}\left(\tau-\lambda_{i} I\right)^{-j}$ so we are coming down to the case $Q=(X-\lambda)^{m}$ with $m \in \mathbb{N}^{*}$ and $|\lambda| \neq 1$.

First case: $|\lambda|>1$ :

$$
(\tau-\lambda I)^{-m}=(-\lambda)^{-m}\left(I-\frac{\tau}{\lambda}\right)^{-m}=(-\lambda)^{-m} \sum_{k=0}^{+\infty} C_{-m}^{k}\left(\frac{-\tau}{\lambda}\right)^{k}
$$

so for $x \in A P(\mathbb{Z}, \mathbb{C})$ one has

$$
(\tau-\lambda I)^{-m} x=y \text { where } y_{n}=\sum_{k=0}^{+\infty} C_{-m}^{k}(-\lambda)^{-k-m} x_{n+k} .
$$

Second case: $|\lambda|<1$

$$
(\tau-\lambda I)^{-m}=\tau^{-m}\left(I-\lambda \tau^{-1}\right)^{-m}=\sum_{k=0}^{+\infty} C_{-m}^{k}(-\lambda)^{k} \tau^{-k-m},
$$

hence for $x \in A P(\mathbb{Z}, \mathbb{C})$ one has

$$
(\tau-\lambda I)^{-m} x=y \text { with } y_{n}=\sum_{k=0}^{+\infty} C_{-m}^{k}(-\lambda)^{k} x_{n-m-k} .
$$

Let $\left(h_{n}\right)_{n \in \mathbb{Z}}$ be bounded. Then we look for a bounded solution for the following difference equation :

$$
\begin{aligned}
& 2 x_{n+4}-13 x_{n+3}+30 x_{n+2}-28 x_{n+1}+8 x_{n}=h_{n} . \\
Q= & 2 x^{4}-13 x^{3}+30 x^{2}-28 x+8=(2 x-1)(x-2)^{3} \\
\frac{1}{Q}:= & \frac{1}{3} \frac{1}{(x-2)^{3}}-\frac{2}{9} \frac{1}{(x-2)^{2}}+\frac{4}{27} \frac{1}{x-2}-\frac{8}{27} \frac{1}{2 x-1} \\
= & g_{1}+g_{2}
\end{aligned}
$$

where

$$
g_{1}:=\frac{-8}{27} \frac{1}{2 x-1}
$$

and

$$
g_{2}:=\frac{1}{3} \frac{1}{(x-2)^{3}}-\frac{2}{9} \frac{1}{(x-2)^{2}}+\frac{4}{27} \frac{1}{x-2} .
$$

For $\frac{1}{2}<|x|<2$, we have

$$
\begin{gathered}
g_{1}=\sum_{n=1}^{+\infty} \frac{a_{n}}{x^{n}}, a_{n}:=-\frac{8}{27} \frac{1}{2^{n}} \\
g_{2}:=\sum_{n=0}^{+\infty} b_{n} x^{n}, b_{n}:=-\frac{1}{48} \frac{1}{2^{n}} n^{2}-\frac{17}{144} \frac{1}{2^{n}} n-\frac{37}{216} \frac{1}{2^{n}} .
\end{gathered}
$$

the solution $x_{n}$ is given by:

$$
x_{k}:=\sum_{n=1}^{\infty} a_{n} h_{k-n}+\sum_{n=0}^{\infty} b_{n} h_{k+n}
$$

$$
\begin{aligned}
x_{k} & :=\sum_{n=1}^{\infty}-\frac{8}{27} \frac{1}{2^{n}} h_{k-n} \\
& +\sum_{n=0}^{\infty}\left(-\frac{1}{48} \frac{1}{2^{n}} n^{2}-\frac{17}{144} \frac{1}{2^{n}} n-\frac{37}{216} \frac{1}{2^{n}}\right) h_{k+n}
\end{aligned}
$$

Let $y \in P A P_{0}(\mathbb{Z}, \mathbb{C})$ and $P \in \mathbb{C}[X]$. We study the existence and uniqueness of solutions in $P A P_{0}(\mathbb{Z}, \mathbb{C})$ of the following equation

$$
P\left(\tau_{0}\right) x=y,
$$

where $\tau_{0}$ is the linear map induced by $T$ on $P A P_{0}(\mathbb{Z}, \mathbb{C})$. Let $x \in P A P_{0}(\mathbb{Z}, \mathbb{C})$ be such that $P\left(\tau_{0}\right) x=0$. From lemma (2.10), we have

$$
\begin{aligned}
& \operatorname{ker} P\left(\tau_{0}\right)=\operatorname{vect}\left(\left\{\left(\lambda_{i}^{n}\right)_{n \in \mathbb{Z}}, i=1, \cdots, r\right\}\right) \cap P A P_{0}(\mathbb{Z}, \mathbb{C}) \\
& \subset A P(\mathbb{Z}, \mathbb{C}) \cap P A P_{0}(\mathbb{Z}, \mathbb{C})=\{0\} .
\end{aligned}
$$

Then we have the uniqueness of the solution.

Remark 3.7: Unlike to the almost periodic case, $x$ bounded and $P\left(\tau_{0}\right) x=y$ is not enough to get that $x \in P A P_{0}(\mathbb{Z}, \mathbb{C})$ In fact, we have the following counter example:

$$
x_{n+1}-x_{n}=2^{-|n|}, n \in \mathbb{Z},
$$

the solutions are given by

$$
x_{n}=\left\{\begin{array}{ll}
x_{0}+\sum_{k=0}^{n-1} 2^{-k}, & \text { if } n>0 \\
x_{0}-\sum_{k=n}^{-1} 2^{-|k|}, & \text { if } n<0
\end{array}, x_{0} \in \mathbb{C} .\right.
$$

Then all solutions are bounded, on the other part one has: $\lim _{n \rightarrow+\infty} x_{n}=x_{0}+2$ and $\lim _{n \rightarrow-\infty} x_{n}=x_{0}-1$. If $x \in P A P_{0}$ we will have $x_{0}+2=x_{0}-1=0$ which is absurd, consequently, the solution is not in $P A P_{0}$.

As a consequence of proposition (2.14), we get the following result.

Proposition 3.8: Let $\lambda$ be a complex number such that $\lambda \neq 0, p \in \mathbb{N}$, and $Q$ a polynomial with degree p. Then

$$
\begin{aligned}
\operatorname{Im}\left(\tau_{0}-\lambda I\right)^{p+1}= & \left\{y \in P A P_{0}(\mathbb{Z}, \mathbb{C})\right. \text {, such that } \\
& \left.\left(Q(n) \lambda^{n}\right)_{n \in \mathbb{Z}} * y \in\left(P A P_{0}\right)_{p, \lambda}\right\} .
\end{aligned}
$$

In particular

$$
\begin{aligned}
\operatorname{Im}\left(\tau_{0}-\lambda I\right)^{p+1}= & \left\{y \in P A P_{0}(\mathbb{Z}, \mathbb{C})\right. \text {, such that } \\
& \left.\left(n^{p} \lambda^{n}\right)_{n \in \mathbb{Z}} * y \in\left(P A P_{0}\right)_{p, \lambda}\right\} .
\end{aligned}
$$




$$
\begin{aligned}
& \operatorname{Im} P\left(\tau_{0}\right)=\left\{y \in P A P_{0}(\mathbb{Z}, \mathbb{C})\right. \text {, such that } \\
& \left.\forall i \in[1, r],\left(n^{m_{i}-1} \lambda_{i}^{n}\right)_{n \in \mathbb{Z}} * y \in\left(P A P_{0}\right)_{m_{i}-1, \lambda_{i}}\right\} .
\end{aligned}
$$

Remark 3.9: In ergodic case, for the calculation of the solutions, the method is similar to the one given in the almost periodic case, firstly we begin with solution of the following equation

$$
\left(\tau_{0}-I\right)^{p+1}(x)=y, x=y * c_{p}-\sum_{0 \leq k \leq p} \alpha_{k} c_{k}
$$

with $\left(\alpha_{k}\right)_{1 \leq k \leq p}$ are determined by equations (8) and (9) but this time the $\alpha_{0}$ is not arbitrarily, but $\alpha_{0}$ is the mean value of $y * c_{p}-\sum_{1 \leq k \leq p} \alpha_{k} c_{k}$, then the existence of solutions needs more $y * c_{p}-\sum_{1 \leq k \leq p} \alpha_{k} c_{k}$ to have a mean value $\alpha_{0}$ then $y * c_{p}-\sum_{0 \leq k \leq p} \alpha_{k} c_{k} \in P A P_{0}$.

Example 3.10: Let $\sum_{k \geq 0} a_{k}$ be an absolutely convergent series, $P \in \mathbb{C}[X],\left(z_{k}\right)_{k \geq 0}$ a family of complex numbers with modulus equal 1, such that $\inf _{k \geq 0}\left|P\left(z_{k}\right)\right|>0$ and $y_{n}=\sum_{k=0}^{+\infty} a_{k} z_{k}^{n}$. Then the following equation

$$
P(\tau) x=y,
$$

has almost periodic solutions. In fact, if we put $x_{n}=\sum_{k=0}^{+\infty} \frac{a_{k}}{P\left(z_{k}\right)} z_{k}^{n}$, one has $x_{n}$ is well defined and

$$
\text { for all } i \geq 0,\left(\tau^{i}(x)\right)_{n}=x_{n+i}=\sum_{k=0}^{+\infty} \frac{a_{k}}{P\left(z_{k}\right)} z_{k}^{n+i}
$$

it results that

$$
(P(\tau) x)_{n}=\sum_{k=0}^{+\infty} \frac{a_{k}}{P\left(z_{k}\right)} P\left(z_{k}\right) z_{k}^{n}=\sum_{k=0}^{+\infty} a_{k} z_{k}^{n}=y_{n}
$$

the equation admits solutions in $A P(\mathbb{Z}, \mathbb{C})$ The hypothesis $\inf _{k \geq 0}\left|P\left(z_{k}\right)\right|>0$ is necessary, as we remark it through the following counter example :

$$
x_{n+1}-x_{n}=\sum_{k=0}^{+\infty} \frac{1}{k^{2}} \exp \left(\frac{i n}{k^{2}}\right) .
$$

If the solution exists, then

$$
a\left(\frac{1}{k^{2}}, x\right)\left(\exp \left(\frac{i}{k^{2}}\right)-1\right)=\frac{1}{k^{2}}
$$

and

$$
\left|a\left(\frac{1}{k^{2}}, x\right)\right| \underset{k \rightarrow+\infty}{\sim} 1
$$

which contradicts the Parsevall's identity, we deduce that the equation does not have a solution.

\section{Application}

More details and the motivation on this applications can be found in [4,14-20] and the references cited therein.

To apply the previous results, we consider the following system

$$
\left\{\begin{aligned}
(p-1) x_{2 n+1}+(1-p) x_{2 n-1} & =\left(\frac{q}{2}-1\right) x_{2 n+2} \\
& +\left(1+3 \frac{q}{2}+p\right) x_{2 n}-p x_{2 n-2}+a_{n} \\
x_{2 n+1}+(1-2 p) x_{2 n-1}=- & (p-q-2) x_{2 n}-p x_{2 n-2}+b_{n}
\end{aligned}\right.
$$

where $a_{n}, b_{n} \in A P(\mathbb{Z}), \quad p, q \in \mathbb{R}$ with $q \neq 0$.

Remark 4.1: The last system comes from the research of solutions of the following second order differential equation with piecewise constant argument:

$$
\frac{\mathrm{d}^{2}}{\mathrm{~d} t^{2}}[x(t)+p x(t-1)]=q x\left(2\left[\frac{t+1}{2}\right]\right)+f(t) .
$$

where [.] denotes the greatest integer function.

In the case where $p \neq 1$, the system has a unique solution $\left(x_{n}\right)_{n \in \mathbb{Z}}$ in $A P(\mathbb{Z})$, here we intend to study the situation where $p=1$, the system becomes

$$
\left\{\begin{array}{l}
0=\left(\frac{q}{2}-1\right) x_{2 n+2}+\left(2+3 \frac{q}{2}\right) x_{2 n}-x_{2 n-2}+a_{n} \\
x_{2 n+1}-x_{2 n-1}=(1+q) x_{2 n}-x_{2 n-2}+b_{n},
\end{array}\right.
$$

or more

$$
\left\{\begin{array}{l}
P(\tau) x_{2 n-2}=2 a_{n} \\
(\tau-I) x_{2 n-1}=((1+q) \tau-I) x_{2 n-2}+b_{n},
\end{array}\right.
$$

where

$$
P(X)=(2-q) X^{2}-(4+3 q) X+2 .
$$

We know that $P(\tau)$ is invertible if and only if the roots of $P$ are with modulus different from 1 .

Proposition 4.2: 1) Let $a_{0}, a_{1}, a_{2} \in \mathbb{R}$. The equation $a_{2} x^{2}+a_{1} x+a_{0}=0$ has roots of modulus 1 if and only if $\left|a_{2}+a_{0}\right|=\left|a_{1}\right|$ or

$$
\left\{\begin{array}{l}
a_{2}=a_{0} \\
\left|a_{1}\right|<2\left|a_{0}\right| .
\end{array}\right.
$$

2) For $a_{3} \in \mathbb{R}^{*}$, the following equation 


$$
a_{3} x^{3}+a_{2} x^{2}+a_{1} x+a_{0}=0,
$$

has roots of modulus 1 if and only if

$$
\left|a_{2}+a_{0}\right|=\left|a_{3}+a_{1}\right|
$$

or

$$
\left\{\begin{array}{l}
a_{0}^{2}-a_{2} a_{0}=a_{3}^{2}-a_{1} a_{3} \\
\left|a_{2}-a_{0}\right|<2\left|a_{3}\right|
\end{array}\right.
$$

Proof. It is clear that \pm 1 are roots of (14) if and only if $\left|a_{2}+a_{0}\right|=\left|a_{1}\right|$ If $a_{1}^{2}<4 a_{2} a_{0}$, the equation admits two conjugate complex roots which are of modulus 1 if and only if their product is equal 1 , which is equivalent to $a_{2}=a_{0}$, the above condition becomes then $\left|a_{1}\right|<$ $2\left|a_{0}\right|$ For the second equation, it admits \pm 1 as roots if and only if $\left|a_{2}+a_{0}\right|=\left|a_{3}+a_{1}\right|$, we can assume that $a_{3}=1$, if not we divide the equation by $a_{3}$, it is a matter to prove that

$$
\left\{\begin{array}{l}
a_{0}^{2}-a_{2} a_{0}=1-a_{1} \\
\left|a_{2}-a_{0}\right|<2
\end{array}\right.
$$

since the equation admits always a real root $r$, it will have non real roots with modulus equal to 1 if and only if it will be factorized as follows

$$
x^{3}+a_{2} x^{2}+a_{1} x+a_{0}=(x-r)\left(x^{2}+c x+1\right) \text { with }|c|<2,
$$

whch implies that $r=-a_{0}$ is a root, in the sequel $\left(-a_{0}^{2}+a_{2} a_{0}-a_{1}+1\right) a_{0}=0$.

If $a_{0}=0$, we obtain $x^{2}+a_{2} x+a_{1}=0$ admits complex roots with modulus equal to 1 then from the previous result, we deduce that

$$
\left\{\begin{array}{r}
a_{1}=1 \\
\left|a_{2}\right|<2
\end{array}\right.
$$

If $a_{0}^{2}-a_{2} a_{0}=1-a_{1}$, the equation can be written as follows

$$
\left\{\begin{array}{l}
x^{3}+a_{2} x^{2}+a_{1} x+a_{0}=x^{3}+a_{2} x^{2}+\left(1-a_{0}^{2}+a_{2} a_{0}\right) x+a_{0} \\
=\left(x+a_{0}\right)\left(x^{2}+\left(a_{2}-a_{0}\right) x+1\right)
\end{array}\right.
$$

We will have no real roots with modulus equal to 1 if and only if $\left|a_{2}-a_{0}\right|<2$.

Corollary 4.3: 1) If $q \neq-4$, the roots of $P$ are of modulus different from 1 (we assume that $q \neq 0$ ).

2) If $q=-4, \quad P(X)=2(X+1)(3 X+1)$.

Proof. It suffices to apply the previous proposition.

Proposition 4.4: If $q \neq-4$, the system (13) admits solutions if and only if

$$
a_{n}-2 b_{n} \in \operatorname{Im}(\tau-I) .
$$

If $q=-4$, the system (13) admits solutions if and only if

$$
\left\{\begin{array}{l}
a_{n} \in \operatorname{Im}(\tau+I) \\
a_{n}-2 b_{n} \in \operatorname{Im}(\tau-I)
\end{array}\right.
$$

Proof. First case: $q \neq-4$ : the system becomes

$$
\left\{\begin{array}{l}
x_{2 n-2}=2 P(\tau)^{-1} a_{n} \\
(\tau-I) x_{2 n-1}=2((1+q) \tau-I) P(\tau)^{-1} a_{n}+b_{n} .
\end{array}\right.
$$

This system admits solutions if and only if

$$
2((1+q) \tau-I) P(\tau)^{-1} a_{n}+b_{n} \in \operatorname{Im}(\tau-I),
$$

or yet

$2((1+q) \tau-I) a_{n}+P(\tau) b_{n} \in \operatorname{Im} P(\tau)(\tau-I)=\operatorname{Im}(\tau-I)$,

since $P(\tau)$ is invertible. Make the Euclidean division of $P$ respectively $(1+q) X-1$ by $X-1$, we see that the previous condition is equivalent to

$$
2 q a_{n}-4 q b_{n} \in \operatorname{Im}(\tau-I),
$$

identically

$$
a_{n}-2 b_{n} \in \operatorname{Im}(\tau-I) .
$$

Second case: $q=-4$ :

The system becomes

$$
\left\{\begin{array}{l}
2(\tau+I)(3 \tau+I) x_{2 n-2}=2 a_{n} \\
(\tau-I) x_{2 n-1}=(-3 \tau-I) x_{2 n-2}+b_{n},
\end{array}\right.
$$

equivalently

$$
\left\{\begin{array}{l}
(\tau+I)(3 \tau+I) x_{2 n-2}=a_{n} \\
x_{2 n-2}=(3 \tau+I)^{-1}\left((I-\tau) x_{2 n-1}+b_{n}\right) .
\end{array}\right.
$$

Let us consider the following system

$$
\left\{\begin{array}{l}
(\tau+I)\left((I-\tau) x_{2 n-1}+b_{n}\right)=a_{n} \\
x_{2 n-2}=(-3 \tau-I)^{-1}\left((\tau-I) x_{2 n-1}-b_{n}\right),
\end{array}\right.
$$

which has solutions if and only if

$$
\left\{\begin{array}{l}
(\tau+I) b_{n}-a_{n} \in \operatorname{Im}(\tau+I)(\tau-I) \\
=\operatorname{Im}(\tau+I) \cap \operatorname{Im}(\tau-I),
\end{array}\right.
$$

which is equivalent to

$$
\left\{\begin{array}{l}
a_{n} \in \operatorname{Im}(\tau+I) \\
a_{n}-2 b_{n} \in \operatorname{Im}(\tau-I) .
\end{array}\right.
$$

\section{Acknowledgements}

The authors would like to thank the referees for their careful reading of the paper. 


\section{References}

[1] C. Corduneanu, "Almost Periodic Discrete Processes," Libertas Mathematica, Vol. 2, 1982, pp. 159-169.

[2] J. Hong and C. Núñez, "The Almost Periodic Type Difference Equations,” Mathematical and Computer Modelling, Vol. 28, No. 12, 1998, pp. 21-31. doi:10.1016/S0895-7177(98)00171-X

[3] S. Elaydi, “An Introduction to Difference Equations,” 3rd Edition, Springer-Verlag, Berlin, 2000.

[4] E. A. Dads and L. Lhachimi, "New Approach for the Existence of Pseudo Almost Periodic Solutions for Some Second Order Differential Equation with Piecewise Constant Argument," Nonlinear Analysis: Theory, Methods and Applications, Vol. 64, No. 6, 2006, pp. 1307-1324. doi:10.1016/j.na.2005.06.037

[5] A. I. Alonso, J. Hong and J. Rojo, “A Class of Ergodic Solutions of Differentiale Quations with Piecewise Constant Arguments," Dynamic Systems and Applications, Vol. 7, 1998, pp. 561-574.

[6] A. M. Fink, "Almost-Periodic Differential Equations, Lecture Notes in Mathematics,” Springer-Verlag, Berlin, Vol. 377, 1974.

[7] S. Zaidman, "Solutions Presque-Périodiques des Équations Différentielles Abstraites," L'Enseignement Mathématique, Vol. 24, No. 1-2, 1978, pp. 87-110.

[8] S. Zaidman, "A Non-Linear Abstract Differential Equation with Almost-Periodic Solution," Rivista di Matematica della Università di Parma, Vol. 10, No. 4, 1984, pp. 331-336.

[9] C. Zhang, "Pseudo Almost Periodic Functions and Their Applications,” Ph.D Thesis, University of Western Ontario, London, Canada, 1992.

[10] C. Zhang, "Pseudo Almost-Periodic Solutions of Some Differential Equations," Journal of Mathematical Analysis and Applications, Vol. 181, No. 1, 1994, pp. 62-76. doi:10.1006/jmaa.1994.1005

[11] C. Zhang, "Integration of Vector-Valued Pseudo Almost Periodic Functions," Proceeding of the American Mathe- matical Society, Vol. 121, No. 1, 1994.

[12] C. Zhang, "A Characterization of Pseudo Almost Periodic Functions in Fourier Analysis," Acta Analysis Functionalis Applicata, Vol. 4, 2002, pp. 110-114.

[13] C. Zhang, "Almost Periodic Type Functions and Ergodicity,” Kluwer Academic Publishers, Dordrecht, 2003.

[14] S. M. Shah and J. Weiner, "Advanced Differential Equations with Piecewise Constant Argument Deviations," International Journal of Mathematics and Mathematical Sciences, Vol. 6, No. 4, 1983, pp. 671-703. doi:10.1155/S0161171283000599

[15] Y. Rong and H. Jialin, "The Existence of Almost Periodic Solutions for a Class of differential Equations with Piecewise Constant Argument," Nonlinear Analysis: Theory, Methods and Applications, Vol. 28, No 8, 1997, pp. 1439-1450. doi:10.1016/0362-546X(95)00225-K

[16] Y. Rong, "Existence of Almost Periodic Solutions of Second Order Neutral Delay Differential Equations with Piecewise Constant Argument," Science in China (Series A), Vol. 41, No. 3, 1998, pp. 232-241. doi:10.1007/BF02879041

[17] R. Yuan, "Pseudo-Almost Periodic Solutions of Second-Order Neutral Delay Differential Equations with Piece-Wise Constant Argument," Nonlinear Analysis: Theory, Methods and Applications, Vol. 41, No. 7-8, 2000, pp. 871-890. doi:10.1016/S0362-546X(98)00316-2

[18] Y. Rong and T. Kupper, "On Quasi-Periodic Solutions of Differential Equations with Piecewise Constant Argument," Journal of Mathematical Analysis and Applications, Vol. 267, No. 1, 2002, pp. 173-193. doi:10.1006/jmaa.2001.7761

[19] Y. Rong, "On a New Almost Periodic Type Solution of a Class of Singularly Perturbed Differential Equations with Piecewise Constant Argument," Science in China (Series A), Vol. 45, No. 4, 2002, pp. 484-502. doi:10.1007/BF02872337

[20] Y. Rong, “The Existence of Almost Periodic Solutions of Retarded Differential Equations with Piecewise Constant Argument," Nonlinear Analysis: Theory, Methods and Applications, Vol. 48, No. 7, 2002, pp. 1013-1032. 